\title{
Vom Arbeitnehmer zum Mitunternehmer? Überschätzte Wirkungen von Kapital- und Gewinnbeteiligungen
}

Gewinn- und Kapitalbeteiligungen der Arbeitnehmer sowie Investivlöhne sind alte Vorschläge, die von ganz unterschiedlichen Seiten immer wieder in die Diskussion gebracht werden. Der Beitrag macht deutlich, dass viele der erhofften Wirkungen illusionär sind. Eine stärkere staatliche Förderung würde zu Fehlanreizen zugunsten relativ riskanter Vermögensanlagen führen. Die Vermögenspolitik sollte das Gesamtvermögen, nicht das Produktivvermögen fokussieren, das nur etwa 15 \% des Vermögens der privaten Haushalte umfasst.

\section{Alte Ideen - neue Illusionen?}

Die Diskussion über Investivlöhne, Kapital- und Gewinnbeteiligungen der Arbeitnehmer, die fast so alt ist wie der Kapitalismus, ist in jüngster Zeit neu entflammt. Viele glauben, dass der anhaltende Rückgang der Lohnquote nicht nur in Deutschland der Globalisierung geschuldet und nur durch Beteiligung der Arbeitnehmer an Kapitalgewinnen korrigierbar sei. Wenn die Gewinne scheinbar unvermeidbar schneller als die Löhne wachsen, müssten die Arbeitnehmer - so die These - zu Mitunternehmern werden, um steigende Ungleichheit mit neuen Konfliktpotenzialen zu vermeiden. Dies ist der Nährboden, der den Ruf nach neuen Anstrengungen zu „Sozialer Kapitalpartnerschaft“ (BDA/BDI 2007) seitens der großen politischen Parteien aufkommen lässt.

In der Bundesrepublik Deutschland blieb die von Ludwig Erhard nach dem Ende des Zweiten Weltkrieges entwickelte Idee der "Teilhabergesellschaft" und des „Volkskapitalismus“ eine Illusion. Jedoch wurde sie in periodisch aufkeimenden Diskussionen immer wieder neu belebt. Nachdem Gewerkschaften und Parteien mehr und mehr von dem Ziel der Veränderung der Eigentumsordnung (z. B. „Vergesellschaftung der Schlüsselindustrien“) Abstand genommen hatten und der „normale Kapitalismus" sich stabilisierte, wurden nach und nach ersatzweise Formen betrieblicher und überbetrieblicher Mitbestimmung sowie staatlich geförderter „Vermögensbildung in Arbeitnehmerhand“
(Vermögensbildungsgesetze) etabliert. Jedoch dominierte die Vermögensbildung der Arbeitnehmer außerhalb des Produktivvermögens. ${ }^{1}$ Beteiligungen der Arbeitnehmer am Produktivvermögen in direkter oder indirekter Form spielen bis heute eine untergeordnete Rolle. Gleichwohl wird von den unterschiedlichsten Seiten - die zunehmende Ungleichheit der Verteilung des Produktivvermögens beklagt. Es wird betont, dass in einigen Ländern, vor allem in den USA, Großbritannien und in Frankreich, der Anteil der Arbeitnehmer am Produktivvermögen größer ist als in Deutschland, insbesondere auch der Anteil am arbeitgebenden Unternehmen. Deutschland hätte Nachholbedarf.

In der aktuellen Situation sind es vor allem die Arbeitgeberverbände, die eine stärkere staatlich geförderte Kapitalbeteiligung (KB) der Arbeitnehmer im Sinne von „Mitarbeiterbeteiligung" (MAB) wünschen. Überbetriebliche Kapitalbeteiligungen in Form von Fonds, wie sie die Gewerkschaften seit jeher fordern, werden abgelehnt. Vorrangig treten die Arbeitgeberverbände aber für „Gewinnbeteiligungen“ (GB) ein. Die Hauptzielsetzung ist die Hoffnung auf höhere betriebliche Produktivität infolge stärkerer Identifikation mit dem Unternehmen.

In der Wissenschaft gibt es eine lange Tradition des Diskurses über Investivlöhne (freilich nur im deutschsprachigen Raum, der Begriff existiert in der angelsächsischen Literatur nicht). Indessen wird im angloamerikanischen Raum mehr über profit related pay und employee ownership diskutiert, d.h. über Gewinn- und Kapitalbeteiligungen der Arbeitnehmer. In der europäischen Fachdiskussion ist von PEPPER die Rede: „Promotion of Employee Parti- cipation in Profits and Enterprise Results" (PEPPER 1991 und 1997).

Im Folgenden soll nach Klärung der verwirrenden Begriffe drei Fragen nachgegangen werden: Warum gibt es in Deutschland ein vergleichsweise geringes $M a ß$ an Kapital- und Gewinnbeteiligungen im Vergleich zu anderen Ländern? Welche wirtschaftswissenschaftlich fundierten Konzeptionen existieren und wie sind diese hinsichtlich der verfolgten Ziele zu bewerten? Kann die Einkommens- und Vermögensverteilung tatsächlich durch Gewinnund Kapitalbeteiligungen der Arbeitnehmer verbessert werden? Im abschließenden Fazit werden die jüngsten Vorschläge von CDU/CSU und SPD kritisch bewertet.

\section{Im Labyrinth der Begriffe}

In der deutschen Diskussion dominiert der Begriff des Investivlohns. Investivlöhne (IL) sind vertraglich vereinbarte Lohnbestand-

\footnotetext{
Unabhängig vom Vermögensbildungsgesetz blieb die staatliche Eigenheimzulage, die 2006 abgeschafft wurde, die mit Abstand finanziell gewichtigste Form der Vermögensförderung, die auch viele Arbeitnehmer in Anspruch nahmen.
}

Jan Priewe, Dr. rer. pol., Professor für Volkswirtschaftslehre an der Fachhochschule für Technik und Wirtschaft (FHTW) Berlin. Arbeitsschwerpunkte: Makroökonomie, Wirtschaftspolitik, Ökonomie von Entwicklungsländern.

e-mail: priewe@fhtw-berlin.de 
teile, die gespart werden. Eigentlich müsste es „Sparlöhne“ heißen, denn „Investieren“ bezieht sich in diesem Zusammenhang auf Geldkapitalbildung, nicht auf Sachanlagen wie in der Volkswirtschaftlichen Gesamtrechnung. IL können zusätzlich zum Barlohn gewährte Entgelte sein, aber es können auch kollektivvertragliche Vereinbarungen zwischen Arbeitgeber und Arbeitnehmern für die Verwendung eines Teils des Barlohns sein. Die Form des Sparens ist bei Investivlöhnen offen: Die Mittel können dem arbeitgebenden Unternehmen als Eigen- oder Fremdkapital zur Verfügung gestellt werden (Mitarbeiter- bzw. Kapitalbeteiligung) oder überbetrieblich in irgendeiner individuell oder kollektiv festgelegten Form gespart werden (z. B. Bausparen, Lebensversicherungen, Wertpapierkäufe, Altersvorsorge in Form von Pensionsfonds).

In Deutschland dominierte jahrzehntelang die Vermögensbildung in Arbeitnehmerhand, die erst mit tarifvertraglichen Vereinbarungen Breitenwirkung erlangte. Die Finanzierung erfolgt durch Arbeitnehmer, Arbeitgeber und Staat (im Rahmen von Einkommensgrenzen), wobei ein relativ breit gefasster Katalog von Anlageformen gefördert wurde. Gewählt wurden ganz überwiegend Lebensversicherungen und Bausparen, zunehmend Altersvorsorgesparen, jedoch selten Anlagen im arbeitgebenden Unternehmen. Ein großer Teil dieser Sparförderung wurde auch als Ansparen für den Kauf dauerhafter Konsumgüter genutzt. In den vergangenen Jahren wurde die staatliche Förderung reduziert.

Der Begriff Investivlöhne (IL) ist problematisch, weil er sich erstens von normalem Sparen der einzelnen Arbeitnehmer (sowie von geförderter „Entgeltumwandlung" zur Altersvorsorge) nicht sinnvoll abgrenzen lässt, zweitens die Zusätzlichkeit zum „Barlohn“ kaum ermittelbar ist und weil drittens suggeriert wird, dass zusätzliche Ersparnisse automatisch zu mehr Investitionen (in Sachkapital bzw. in Produktivvermögen) führen.

Zunehmend setzte sich der Begriff $\mathrm{Ka}$ pital- und Gewinnbeteiligung durch. Kapitalbeteiligungen $(\mathrm{KB})$ liegen demnach vor, wenn Arbeitnehmer dem arbeitgebenden Unternehmen oder anderen Unternehmen Eigen- oder Fremdkapital (oder Mischformen davon) zur Verfügung stellen. MAB bedeutet, dass sich Mitarbeiter mit selbst finanziertem Eigenkapital an ,ihrem“ Un- ternehmen beteiligen, also Miteigentümer werden. In der Folge partizipieren sie dann auch an den Gewinnen des Unternehmens. ${ }^{2} \mathrm{~KB}$ bezieht sich also auf die Beteiligung am Produktivvermögen, d.h. auf das Risikokapital der Unternehmen, wenn man Fremdkapital einmal ausklammert. Bei KB kauft der Arbeitnehmer Kapitalanteile von Unternehmen. Dabei kann der Arbeitgeber dies durch preisliche Vergünstigungen, Optionsscheine etc. unterstützen oder den Arbeitnehmer direkt mit Beteiligungen „entlohnen“.

Bei Gewinnbeteiligungen (GB) erhalten Arbeitnehmer zusätzlich zum festen Lohn gewinnabhängige Entgeltbestandteile. Die „Gewinnbeteiligung“ ist Teil des Lohnes; sie ist ein variabler Lohnbestandteil, also Kostenfaktor, kein Gewinn. Andernfalls wäre die Unterscheidung von Lohn und Gewinn überflüssig. Insofern ist der Begriff irreführend. Wäre es eine echte Gewinnbeteiligung der Arbeitnehmer, ergäbe sich eine Gewinn- und Renditeminderung der Kapitaleigner, für die diese keinen Anreiz hätten. Es wäre daher eigentlich sinnvoller, von profit-related pay oder variabler Entlohnung zu sprechen. GB können auch in Form von KB am arbeitgebenden Unternehmen gewährt werden (Belegschaftsaktien, Aktienoptionen), wie es bei der Entlohnung des Managements in Großunternehmen verbreitet ist. Dies wäre eine Mischform, eine Schnittmenge von $\mathrm{GB}$ und $\mathrm{KB}$. Eine andere Mischform liegt vor, wenn GB einbehalten und in anderen Unternehmen oder in einem vom arbeitgebenden Unternehmen gemanagten Fond (z. B. ein Pensionsfond) angelegt werden („deferred profit sharing“, weit verbreitet in den USA).

Grundsätzlich sind KB und GB - von den Mischformen abgesehen - unterschiedliche Sachverhalte, auch wenn sie häufig unter dem irreführenden Oberbegriff der Mitarbeiterbeteiligung („financial participation") zusammengefasst werden.

\section{Geringe Kapital- und Gewinnbeteiligungen in Deutschland}

In Deutschland gibt es traditionell nur ein geringes $\mathrm{Ma} ß$ an $\mathrm{KB}$ am arbeitgebenden Unternehmen. Die Arbeitsgemeinschaft für Partnerschaft in der Wirtschaft (AGP
2007) ermittelte für 2006 ca. 3.600 Beteiligungsunternehmen mit 2 Mio. beteiligten Mitarbeitern bei einem Beteiligungskapital von durchschnittlich $6.135 €$ je Mitarbeiter und insgesamt 12,3 Mrd. $€$ Beteiligungskapital. Im Jahre 2005 existierten MAB nur in etwa $2 \%$ aller Betriebe (ab einer Betriebsgröße von einem Mitarbeiter, Bellmann/Möller 2006a) und für ca. 5,7 \% aller Arbeitnehmer. Der Anteil des Beteiligungsvermögens der Arbeitnehmer - bezogen auf MAB - am gesamten Produktivvermögen ist verschwindend gering, er bewegte sich 1999 in der Größenordnung von 0,6 \% (Priewe 2000, S. 39). Über indirekte KB der Arbeitnehmer - im Wesentlichen Aktienvermögen im Rahmen der Geldvermögensbildung der privaten Haushalte gibt es keine genauen Angaben. ${ }^{3} 69$ \% der Arbeitnehmer mit Mitarbeiterbeteiligung besitzen Belegschaftsaktien, das betrifft knapp $17 \%$ der Beteiligungsunternehmen. 2006 ist die Zahl der Belegschaftsaktionäre auf knapp 1,1 Mio. gesunken (DAI 2007), das sind etwa 3,1\% aller Arbeitnehmer. Dabei muss berücksichtigt werden, dass Aktiengesellschaften nur 0,2\% aller Unternehmen in Deutschland ausmachen; hier arbeiten aber 2,5 Mio. Personen, d.h. rund $8 \%$ aller Arbeitnehmer. Stille Beteiligungen existieren in ca. 1.000 Unternehmen mit einem Wert von nur etwa 1,3 Mrd. $€$. Genussrechte, Mitarbeiterdarlehen und Ähnliches spielen eine marginale Rolle (AGP 2007).

Der geringen Bedeutung von Mitarbeiterbeteiligung (MAB) steht die große Verbreitung der vom Arbeitgeber sowie vom Staat geförderten vermögenswirksamen Leistungen gegenüber, die durch tarifvertragliche Vereinbarungen zustande kam: Mitte der 1990er Jahre erhielten $95 \%$ aller westdeutschen und $63 \%$ aller ostdeutschen tarifgebundenen Arbeitnehmer vermögenswirksame Leistungen. Diese flossen nur zu etwa $10 \%$ in Risikokapital (Tofaute 2006, S. 81).

\footnotetext{
2 Diese Gewinne beruhen jedoch auf Eigentumsanteilen und dürfen nicht mit "Gewinnbeteiligun gen" im Sinne gewinnabhängiger Entlohnung verwechselt werden

32006 besaßen 12 \% der deutschen Bevölkerung mit deutlich rückläufiger Tendenz - Aktien oder Aktienfondsanteile, die Zahl der direkten Aktionäre (Besitz von Aktien ohne Fonds) lag bei 5,3\% der Bevölkerung. Etwa ein Viertel der Aktienbesitzer (ohne Fondsbesitzer) sind Belegschaftsaktionäre (DAI 2006).
} 
Nach Angaben des Instituts für Arbeitsmarkt- und Berufsforschung (IAB) existieren im Jahr 2005 in rund $9 \%$ aller Betriebe Gewinnbeteiligungen (GB) in Form erfolgsabhängiger Zuwendungen (Bellmann/ Möller 2006a), in Unternehmen mit mehr als 500 Mitarbeitern ist es ein Drittel. Dies betrifft ca. 3,8 Mio. Beschäftigte (Schnabel 2004, S. 82), rund $12 \%$ aller Arbeitnehmer. Inzwischen dürfte es jedoch in erheblich größerem Stil erfolgsbezogene Entgeltsysteme geben, da in den letzten Jahren in vielen Branchen erfolgsabhängige Sonderleistungen tarifvertraglich vereinbart wurden, häufig in der Größenordnung von bis zu einem halben Monatsgehalt.

Für den internationalen Vergleich gibt es kaum verlässliche Daten (Hardes/Wickert 2000; Hofmann/Holzner 2002; Schnabel 2004; Blum 2006; Priewe/Havighorst 1999). Eine vielfach herangezogene neuere Studie von Pendleton et al. (2001) mit Daten für 1999/2000 zeigt, dass Deutschland bei den $\mathrm{KB}$ und zu einem geringeren Maße bei den GB im unteren Mittelfeld von 14 europäischen Ländern liegt. Die Angaben beziehen sich auf Betriebe, nicht auf Beschäftigte. In die Umfrage wurden nur Betriebe mit mehr als 200 Beschäftigten aufgenommen, in denen $\mathrm{KB}$ und GB für mehr als $50 \%$ der Belegschaft gelten. Deutschland liegt demnach mit $10 \%$ der Betriebe mit Aktienbeteiligungen deutlich unter dem europäischen Durchschnitt (16\%), ebenso bei den Betrieben mit Gewinnbeteiligungen mit $18 \%$ gegenüber einem Durchschnitt von $36 \%$ (Pendleton et al. 2001, S. 31 und 34).

Frankreich und die Niederlande liegen entsprechend dieser Studie bei GB mit großem Abstand vor den anderen Ländern; $\mathrm{KB}$ in Form von Aktienbeteiligungen sind in Großbritannien, Frankreich und den Niederlanden deutlich überdurchschnittlich verbreitet. Bezieht man die USA ein, dann zeigen sich dort wesentlich höhere Werte für $\mathrm{KB}$, weniger für $\mathrm{GB}$.

Die wichtigsten Gründe für die relativ wenig verbreiteten Kapitalbeteiligungen und auch Gewinnbeteiligungen in Deutschland sind die Folgenden:

- Das umlagefinanzierte staatliche Rentensystem mit einer schwachen zweiten Säule der Betriebsrenten erklärt den Unterschied bei KB zu den USA und Großbritannien. Für Arbeitnehmer ist das deutsche Rentensystem zweifellos dem amerikanischen, das auf Kapitaldeckung beruht, überlegen (DRV 2005).
- Die Dominanz von Flächentarifverträgen mit traditionell starken Branchengewerkschaften - gegenüber jenen Ländern, die eine starke Verbreitung von KB und GB aufweisen - hat einer betriebsbezogenen und damit ungleichen Entlohnung eine randständige Bedeutung zugewiesen. Stattdessen wurde eine an der gesamtwirtschaftlichen Produktivität orientierte Lohnpolitik gefördert. Erst in einer Zeit gewerkschaftlicher Schwächung, vor allem infolge hoher Arbeitslosigkeit, wurden gewinnabhängige variable Entgeltformen in vielen Tarifverträgen in größerem Stil akzeptiert, allerdings blieb der variable Anteil bislang gering.

- Die Struktur deutscher Unternehmen weist traditionell einen hohen Anteil von Personengesellschaften auf, in denen Beteiligungen von Arbeitnehmern rechtlich schwierig sind. Zudem steht die Mehrzahl der Eigentümer von Klein- und Mittelbetrieben Arbeitnehmerbeteiligungen skeptisch gegenüber.

- Die Gewerkschaften und die Mehrzahl der Arbeitnehmer sind risikoavers und bevorzugen andere Vermögensanlagen. Überbetriebliche Kapitalbeteiligungen an Kapitalfonds, von den Gewerkschaften favorisiert, wurden von den Arbeitgebern abgelehnt, betriebliche von den Gewerkschaften. Nur dort, wo attraktive oder gegen Risiken abgesicherte Beteiligungsformen existieren (z. B. günstige Kaufpreise für Belegschaftsaktien solider und langfristig profitabler Großunternehmen auf der "Sonnenseite“ des Strukturwandels), finden Arbeitnehmerbeteiligungen statt, von eher seltenen Notsituationen (z. B. Sanierungsfälle) abgesehen.

- Die staatliche Förderung hat die allgemeine Vermögensbildung favorisiert, in jüngster Zeit zwar die MAB und die Altersvorsorge etwas günstiger gestellt, jedoch wurde die Eigenheimförderung wie auch die Sparförderung insgesamt (Sparerfreibetrag) reduziert. In anderen Ländern wurde die Risikokapitalbeteiligung von Arbeitnehmern staatlich gefördert oder sogar den Unternehmen gesetzlich vorgeschrieben (Frankreich).

Insofern kann von einem deutschen Nachholbedarf bei KB und GB überhaupt keine Rede sein, da die Systeme der Altersvorsorge, die Struktur der Rechtsformen der Unternehmen sowie die Lohnfindungssysteme zwischen den OECD-Ländern viel zu unterschiedlich sind - es sei denn, man will
KB und GB nutzen, um so die Altersversorgung in stärkerem Maße auf das Kapitaldeckungsprinzip umzustellen und Flächentarifverträge aufzuweichen.

\section{Alternative Konzeptionen und Ziele}

Kapital- und Gewinnbeteiligungen sollen, den Verlautbarungen ihrer Befürworter folgend, einer Vielzahl von Zielen dienen, vor allem: Produktivitätssteigerung durch mehr Motivation und Identifikation der Mitarbeiter mit dem Unternehmen; Verbesserung der Einkommens- und Vermögensverteilung zugunsten der Arbeitnehmer; gesellschaftspolitischen Zielen wie „partnerschaftliche Unternehmenskultur“ oder gerechtere Verteilung des Produktivvermögens; höhere Investitionen und mehr Beschäftigung; bessere Altersvorsorge. Häufig sind die verfolgten Ziele aber diffus. GB und KB werden häufig per se positiv als „Allzweckwaffe“ bewertet. Behauptungen ersetzen dabei allzu oft genauere Wirkungsanalysen (Priewe/Havighorst 1999, S. 44-54).

\subsection{BETRIEBLICHE PRODUKTIVITÄTS- STEIGERUNGEN?}

Das am häufigsten genannte Ziel von $\mathrm{KB}$, aber auch von GB, ist die betriebliche Produktivitätssteigerung durch höhere Motivation der Mitarbeiter. Hierzu wurden Dutzende empirischer Studien durchgeführt, die insgesamt zu nicht eindeutigen Ergebnissen führen (Blum 2006; Schnabel 2004; Hofmann/Holzner 2002; Bellmann/Möller 2006a; Wolf/Zwick 2002). Zwar ist meist eine Korrelation von KB/GB und Produktivitätswachstum feststellbar, aber die Kausalität ist nicht evident. Häufig sind es gerade produktivitätsstarke, überdurchschnittlich wachsende Betriebe, die ihre Mitarbeiter am Kapital beteiligen oder erfolgsabhängig entlohnen. Die Ursachen für die Produktivitätsdynamik können vielfältig sein und wenig mit $\mathrm{KB} / \mathrm{GB}$ zu tun haben. Ebenso sind die Motive der Betriebe, KB oder GB einzuführen, sehr unterschiedlich. Da KB und GB nicht an individuelle Leistungen, sondern an das Betriebs- bzw. Unternehmensergebnis insgesamt gebunden sind, dürften sogar Fehlanreize für individuelles Trittbrettfahrer-Verhalten gege- 
ben sein. Die meisten Studien zeigen, dass Produktivitätssteigerungen bei KB/GB nur auftreten, wenn eine leistungsorientierte Unternehmensorganisation und ein entsprechendes Personalmanagement vorliegen. $\mathrm{KB} / \mathrm{GB}$ sind dabei ein mögliches, aber kein zwingend notwendiges Element. Schließlich ist darauf hinzuweisen, dass betriebliche Produktivitätssteigerungen auch mit anderen Mitteln erreichbar sind, insbesondere durch Flächentarifverträge mit Lohnsteigerungen, die sich an gesamtwirtschaftlichen Produktivitätszuwächsen orientieren: Unterdurchschnittlich produktive Betriebe werden mit der „Lohnpeitsche " zu Innovationen getrieben, überdurchschnittlich produktive werden mit nur durchschnittlich steigenden Löhnen „belohnt“. Insofern ist die Begründung von KB/GB mit Produktivitäts- und Motivationsanreizen anfechtbar. Richtig ist sicher, dass Beteiligungen zu einer „partnerschaftlichen Unternehmenskultur" beitragen können. Allerdings ist dies eine sehr diffuse Vision, die vielfältig interpretierbar ist.

\subsection{GERECHTERE VERTEILUNG DES PRODUKTIVVERMÖGENS?}

Ein anderes, häufig angeführtes Ziel von Kapitalbeteiligungen ist die „gerechtere Verteilung des Produktivvermögens". Aber ist dieses Ziel ein Selbstzweck? Häufig wird davon ein gesellschaftlicher Machtausgleich und stärkerer Einfluss von Arbeitnehmern und Gewerkschaften auf wirtschaftliche Entscheidungen erwartet. Allerdings bleiben derartige Vorstellungen meist sehr vage. Nach welchen Gesichtspunkten sollen unternehmensbezogene Entscheidungen gefällt werden, wenn die Belegschaften nennenswerte Beteiligungen am arbeitgebenden Unternehmen haben? Für die Arbeitnehmer würde eine in großem Stil existierende Mitarbeiterbeteiligung am „eigenen“ Unternehmen in der Regel eine riskante Vermögensanlage bedeuteten, da sie durch eine geringe Diversifikation des Arbeitnehmer-Vermögens, eine Kombination von Arbeitsplatz- und Anlagerisiko sowie durch einen Zielkonflikt zwischen Lohn- und Vermögenspolitik gekennzeichnet wäre. Überbetriebliche Fonds zur Anlage in Produktivvermögen haben gegenüber Mitarbeiterbeteiligungen am arbeitgebenden Unternehmen zwar den Vorteil größerer Diversifikation, aber es wäre immer noch eine Anlage in Risikokapital. Wie oben erwähnt, bevorzugen Arbeitnehmer, besonders in Deutschland, bei ihren Sparentscheidungen weniger riskante Anlagen in Geldvermögen und Immobilien. Es bedürfte schon sehr starker Anreize, um die Präferenzen in Richtung Produktivvermögensbeteiligung zu ändern. In der Regel geht dies nur mit massiver staatlicher Förderung. Kapitalgedeckte Altersvorsorgesysteme könnten zwar auf längere Sicht eine substanzielle Beteiligung der Arbeitnehmer am Produktivvermögen ermöglichen, aber sie werden durch erhöhte Risiken erkauft. Selbst in diesem Fall wäre die Altersvorsorge das Ziel, nicht die veränderte Produktivvermögensverteilung. Insofern ist es an der Zeit, das Ziel einer gerechteren Verteilung des Produktivvermögens zugunsten einer gerechteren Vermögensverteilung insgesamt aufzugeben, die Risikokapital ebenso wie andere Vermögensformen umfasst und im Übrigen nicht nur Arbeitnehmer einbezieht. Das Produktivvermögen macht nur etwa $15 \%$ des Vermögens der privaten Haushalte aus. ${ }^{4}$

Andere Konzeptionen argumentieren, dass durch stärkeres Sparen der Arbeitnehmer zwecks Beteiligung am Produktivvermögen auf betrieblicher oder überbetrieblicher Ebene die Einkommens- und Vermögensverteilung verbessert werden könne. Natürlich ist es zutreffend, dass gesparte, also nicht konsumierte Lohneinkommen zusätzliches Einkommen in Form von Zinsen, Dividenden, Unternehmensgewinnen etc. generieren. Dies impliziert aber längerfristiges Sparen und schließt Ansparen für den Kauf dauerhafter Konsumgüter und nachfolgendes Entsparen aus. Risikoorientierte Anlagen, insbesondere in Produktivvermögen, versprechen höhere Renditen als Anlagen mit geringem Risiko. Bei Einkommensgruppen mit geringem Vermögen und risikobehaftetem Einkommen (Arbeitsplatzverlust) ist Risikoaversion ebenso rational wie größere Risikobereitschaft bei Vermögenden. Insofern ist es geradezu irrational, Arbeitnehmern und Gewerkschaften mangelnde Risikobereitschaft vorzuwerfen (Sievert 1992, S. 24). Trotzdem bleibt das Argument, dass eine höhere Arbeitnehmer-Sparquote die Einkommens- und Vermögensverteilung zugunsten der Arbeitnehmer-Haushalte verändert. Dies gilt insbesondere dann, wenn die Realzinsen über den Reallohnzuwächsen liegen, wie es seit Langem in Deutschland der Fall ist. Wenn vermutet wird, dass Arbeitnehmer (oder andere Be- völkerungsgruppen) eher kurzsichtig-konsumorientiert handeln und längerfristige Sparziele im individuellen Kalkül vernachlässigen, kann es sinnvoll sein, dass der Staat und/oder die Tarifparteien das Sparen - insbesondere bei unteren Einkommensschichten - fördern. Gerade hier ist aber von Anlagen in Risikokapital abzuraten, gleichgültig ob im arbeitgebenden Unternehmen oder in überbetrieblichen Fonds.

\subsection{NEOKLASSIK - MEHR SPAREN, HÖHERE INVESTITIONEN, MEHR BESCHÄFTIGUNG?}

Aus neoklassischer Sicht wird zusätzliches Sparen (sowohl von Arbeitnehmern wie von anderen Gruppen) automatisch zu höheren Investitionen, höherem Wachstum und mehr Beschäftigung führen. Es werden sinkende Zinsen erwartet, die mehr Investitionen rentabel machen. In der Phase einer sinkenden Konsumquote und einer ansteigenden Sparquote gibt es zwar eine geringere Konsumgüterproduktion, der jedoch eine stärkere Investitionsgüterproduktion entgegenwirkt. Letztere ist notwendig, da vermehrtes Sparen als vermehrter zukünftiger Konsum verstanden wird. Diese Argumentation ist vor allem deswegen fragwürdig, weil die zinssenkende Wirkung des Sparens die Geldpolitik der Zentralbank ausklammert, weil die Investitionen nicht nur vom Zinssatz abhängen und weil die Konsumsenkung einen Rückgang des Nationaleinkommens zur Folge haben kann, wodurch das gesamtwirtschaftliche Sparen wieder vermindert wird. Überzeugender ist die postkeynesianische

4 Das Deutsche Institut für Wirtschaftsforschung (DIW) hat für das Jahr 2002 berechnet, dass das Nettovermögen je Einwohner in Deutschland ca. $69.000 €$ betrug, davon entfielen zwei Drittel auf Immobilien, 21,8 \% auf Geldvermögen, das Aktien und Investmentfonds einschließt, und 11,7\% auf Sach- und Betriebsvermögen (Sachvermögen umfasst hier Schmuck, Gold, Sammlungen etc.) (eigene Berechnungen nach Krause/Schäfer 2005). Auf Aktien, Investmentfonds und "sonstige Beteiligungen " entfällt nur ein Viertel des Geldvermögens (Sprenger 2007 für das Jahr 2006). Im Durchschnitt der Jahre 1992 bis 2002 machte das Produktivvermögen privater Haushalte nur 15\% ihres Gesamtvermögens aus (Ammermüller et al. 2005, S. 83). Nahezu zwei Drittel des Netto-Gesamtvermögens konzentriert sich dabei auf die oberen $10 \%$ der privaten Haushalte (Krause/ Schäfer 2005, S. 201) 
Sicht: Vermehrte Investitionen ziehen höhere Produktion, höheres Volkseinkommen und damit vermehrtes volkswirtschaftliches Sparen (sei es der Unternehmen, sei es der privaten Haushalte) nach sich. Im Übrigen ist ein positiver Zusammenhang zwischen Kapital- oder Gewinnbeteiligungen und Beschäftigung empirisch zweifelhaft, weshalb eine OECDStudie von staatlicher Förderung abrät (OECD 1995, S. 160 f.).

\subsection{KREISLAUFTHEORIE DER VERTEILUNG - HÖHERES ARBEITNEHMERSPAREN, HÖHERE LOHNQUOTE?}

Die interessanteste Begründung für Investivlöhne (IL) (im Sinne von zusätzlichen „Sparlöhnen“) stammt aus der Kreislauftheorie der Verteilung, die vor allem auf Nicholas Kaldor und Michal Kalecki zurückgeht. Es wird argumentiert, dass eine höhere Sparquote der Arbeitnehmer (unabhängig von der Art der Sparanlage) zu einer höheren Lohnquote führen kann (Oberhauser 2006). Die Sparlöhne können sich auf KB im arbeitgebenden Unternehmen, in anderen Unternehmen, aber auch auf ganz andere Sparformen beziehen. Es handelt sich um eine spezielle Form von GB, die zusätzlich zum „Festlohn“ mit einer Bindung für Sparziele vereinbart wird. Die Zusatzentgelte sollen über produktivitätsorientierte Lohnerhöhungen hinausgehen, weil ansonsten nachfragebedingt ein Rückgang des Inlandsproduktes befürchtet wird. Die „Sparlöhne“ hätten keine inflationstreibende Wirkung, da sie ja die Güternachfrage nicht erhöhen. Neben der Einkommensverteilung wird auch die Vermögensverteilung verbessert. Das zugrunde liegende Modell geht von gegebenem Volkseinkommen aus und unterstellt in der einfachsten Form bei gesamtwirtschaftlicher Betrachtung Lohnkosten als die einzigen Kosten der Unternehmen. Löhne sind demnach nicht nur Kosten, sondern auch Einnahmen der Unternehmen, wenn sie im Inland konsumiert werden. Weitere Einnahmen der Unternehmen stammen definitorisch aus Investitionen (also Käufe von Investitionsgütern I), Konsumausgaben der Unternehmer-Haushalte $\left(\mathrm{C}_{\mathrm{Q}}\right)$, positiven Nettoausgaben des Staates (Nettoneuverschuldung, G-T) sowie aus Exportüberschüssen (Ex-Im). Ceteris paribus vermindert höheres Sparen der Arbeitnehmer $\left(\mathrm{S}_{\mathrm{w}}\right)$ die Ein- nahmen der Unternehmen und damit deren Gewinne (Q). ${ }^{5}$

$\mathrm{Q}=\mathrm{I}+\mathrm{C}_{\mathrm{Q}}+\mathrm{G}-\mathrm{T}+\mathrm{Ex}-\mathrm{Im}-\mathrm{S}_{\mathrm{w}}$

Für die Gewinnquote, den Anteil der Gewinne $\mathrm{Q}$ am Nationaleinkommen Y, gilt also:

$$
\begin{aligned}
\mathrm{Q} / \mathrm{Y}= & \left(\mathrm{I}+\mathrm{C}_{\mathrm{Q}}\right) / \mathrm{Y}+(\mathrm{G}-\mathrm{T}) / \mathrm{Y} \\
& +(\mathrm{Ex}-\mathrm{Im}) / \mathrm{Y}-\mathrm{S}_{\mathrm{w}} / \mathrm{Y}
\end{aligned}
$$

Eine höhere Ersparnis der Arbeitnehmer reduziert also die Profitquote und steigert die Lohnquote. ${ }^{6}$ Die Argumentation ist schlüssig, hat aber drei Schwachstellen. Erstens ist nicht sicher, ob die Arbeitnehmer dank der Investivlöhne wirklich zusätzlich sparen, denn sie könnten ja auch ihr bisheriges normales Sparen reduzieren und damit mehr konsumieren. Zweitens kommt es zum Entsparen mit entgegenwirkenden Verteilungseffekten, wenn Arbeitnehmer nach einiger Zeit des Ansparens ihre Ersparnisse zum Kauf von dauerhaften Konsumgütern verwenden. Drittens ist nicht sicher, ob die Investitionen konstant bleiben, weil die Zusatzentgelte kostenwirksam sind. Zwar könnte ein Teil der Investitionen nun aus Arbeitnehmersparen finanziert werden und in geringerem Maße aus Gewinnen, aber die Kapitalrentabilität würde sinken. Wenn die Investitionen sinken, sinken auch Produktion, Nationaleinkommen und Beschäftigung. Auch die Gewinnquote sinkt; die Arbeitnehmer würden ihre verbesserte Verteilungsposition mit Arbeitslosigkeit bezahlen. Eventuell könnte die Zentralbank die Investitionstätigkeit mit niedrigeren Zinsen stützen, sofern keine Inflationsrisiken bestehen. Beziehen sich die IL in Form von GB vorrangig auf Unternehmen mit überdurchschnittlicher Geschäfts- und Gewinnentwicklung, bei denen kein Einbruch der Investitionstätigkeit $\mathrm{zu}$ erwarten ist, kann das Konzept aufgehen.

\subsection{VARIABLE ARBEITNEHMER- ENTGELTE ZUR BESCHÄFTIGUNGS- SICHERUNG UND EINKOMMENSSTEIGERUNG?}

Von Gewinnbeteiligung im Sinne variabler Entgeltsysteme werden einerseits, wie erwähnt, betriebliche Produktivitätszuwächse erwartet, andererseits wird aber auch eine Beschäftigungsstabilisierung erhofft, vor allem durch flexible allgemeine Lohn- kostensenkung in Rezessionen. Abgesehen von den Produktivitätszielen stehen also Beschäftigungswirkungen im Vordergrund. GB führen jedoch zu einer prozyklischen Nominal- und auch Reallohnentwicklung, von der prozyklische Kosten-, Nachfrageund Preisniveaueffekte ausgehen, die makroökonomisch destabilisieren. Es können sogar deflationäre Folgen eintreten, wie z. B. während der jüngsten Deflationsphase in Japan. Wenn Arbeitskräfte in einer Rezession nicht benötigt werden, weil es an Absatzchancen mangelt, werden auch niedrigere Löhne Entlassungen nicht verhindern können. Aus neoklassischer Sicht könnte eingewendet werden, dass flexible Reallohnanpassungen nach unten in schwachen gesamtwirtschaftlichen Phasen zu steigender Arbeitsnachfrage führen. Dies impliziert, dass die Faktorpreisrelation kurzfristig die Arbeitsnachfrage steuert, sodass Unternehmen bei gegebenem Kapitalstock kurzfristig mehr Arbeit einsetzen, wenn diese relativ zu den Kapitalkosten billiger wird. Zudem muss die Elastizität der Arbeitsnachfrage in Bezug auf den Reallohn größer als eins sein, damit ein konjunkturstimulierendes Wachstum der Lohnsumme und damit der Konsumnachfrage zustande kommt. Häufig werden auch Realkasseneffekte (infolge steigender realer Geldmenge) unterstellt, die bei gegebener nominaler Geldmenge via Lohnund Preissenkungen zu mehr Konsum und Investitionen führen sollen. All dies ist an sehr spezielle Bedingungen geknüpft und außerordentlich wirklichkeitsfremd. In der Realität ist die Faktorkombination von Arbeit und Kapital kurzfristig - d.h. bei gegebenem Kapitalstock und gegebener Technologie - relativ starr, und Realkasseneffekte spielen keine relevante Rolle. Zur beschäftigungssichernden Funktion von GB gibt es nur wenige empirische Untersuchungen, die jedoch nicht zu eindeutigen Ergebnissen führen (u.a. OECD 1995; Gerlach 1997; Schäfer/Rürup 1998).

Ob variable Entgeltsysteme die Einkommensverteilung verändern, hängt maßgeblich von der Höhe der Festlöhne ab. Aus

\footnotetext{
5 Die Gleichungen sind aus der Verwendung bzw. Verteilung des Inlandsproduktes abgeleitet: $Y=C$ $+\mathrm{I}+\mathrm{G}+$ Ex-Im und $\mathrm{Y}=\mathrm{W}+\mathrm{Q}+\mathrm{T}$. W ist die dabei volkswirtschaftliche Lohnsumme, $T$ sind Steuern.

6 Ob dies dauerhaft so bleibt, hängt davon ab, wie die Arbeitnehmer in Zukunft die Vermögenseinkommen verwenden.
} 
gewerkschaftlicher Sicht sollen erfolgsabhängige Entgelte „on top“ gezahlt werden, möglichst zusätzlich zur produktivitätsorientierten Lohnpolitik; aus Arbeitgebersicht sollen sie Bestandteil der Tarifentgelte sein und gegenüber den traditionellen Festlohnentgelten ein Abweichen nach unten wie nach oben ermöglichen (BDA/BDI 2007). Damit hängen die Einkommensveränderungen, die sich am Ende einstellen, von der jeweiligen Verhandlungsstärke der Tarifparteien, von der konjunkturellen Situation und von der Art des sektoralen Strukturwandels ab. Aus der Sicht ihrer Befürworter werden mit GB bzw. variabler Entlohnung auch keine Umverteilungsziele verfolgt (allenfalls indirekt über erhoffte Beschäftigungseffekte).

\section{Fazit}

Insgesamt führt der Überblick über die langjährige Diskussion über Investivlöhne, Kapital- und Gewinnbeteiligungen zu einem ernüchternden Ergebnis. Als Fazit lässt sich resümieren:

- Im Vergleich zu den USA, Frankreich, Großbritannien und den Niederlanden gibt es in Deutschland ein geringeres Maß an Kapitalbeteiligungen der Arbeitnehmer. Dies liegt wesentlich am umlagefinanzierten staatlichen Rentensystem, an der Dominanz des Flächentarifvertrages bei der Lohnbildung sowie an einer staatlichen Vermögenspolitik, die - aus guten Gründen - der allgemeinen Vermögensbildung gegenüber der Fokussierung auf Produktivvermögen Vorrang gibt. Gewinnbeteiligungen im Sinne variabler Entgeltformen haben in den letzen Jahren in Deutschland stark zugenommen.

- Kapitalbeteiligungen von Arbeitnehmern am Produktivvermögen sind riskanter als viele andere Vermögensanlagen, insbesondere wenn es sich um Beteiligungen am arbeitgebenden Unternehmen handelt. Im Kalkül der Arbeitnehmer haben sie gegenüber anderen Sparformen eine untergeordnete Bedeutung, insbesondere wenn es um Altersvorsorge geht. Dies gilt vor allem für Arbeitnehmer mit geringem Einkommen und Vermögen.

- GB und KB sollen zwar vielen Zielen dienen, in Wirklichkeit erreichen sie aber nur wenige. Vor allem ist ihre motivations- und produktivitätssteigernde Wirkung unsicher. Beschäftigungssichernde Wirkungen sind empirisch nicht nachgewiesen. Eine stärkere Beteiligung der Arbeitnehmer am Produktivvermögen könnte - wie in den USA - über kapitalgedeckte Altersvorsorgesysteme (im arbeitgebenden Unternehmen oder in anderen Unternehmen) erreicht werden, ist aber riskant und im Interesse einer Portfoliodiversifizierung nur mit Vorsicht und Zurückhaltung zu empfehlen. Illusionär ist die Hoffnung, durch starke Produktivvermögensbeteiligung von Arbeitnehmern die Machtstruktur im Unternehmenssektor zu ändern; geeignete Konzeptionen sind weit und breit nicht $\mathrm{zu}$ sehen.

- Nicht nur die Lohnhöhe, sondern auch die Lohnverwendung hat Einfluss auf die Einkommensverteilung zwischen Kapital und Arbeit. Eine höhere Sparquote der Arbeitnehmer erhöht ceteris paribus die Lohnquote und lässt vermehrt Vermögenseinkommen in Arbeitnehmerhaushalte fließen. Der Verteilungsspielraum der Arbeitnehmer kann vergrößert werden, wenn über produktivitätsorientierte Lohnzuwächse hinaus „Investivlöhne“ (= Sparlöhne) durchgesetzt werden können, sofern dadurch die Investitionstätigkeit der Unternehmen nicht beeinträchtigt wird und die Sparquote der Arbeitnehmer insgesamt steigt.

- KB von Arbeitnehmern haben einen positiven Einfluss auf die Lohnquote (und längerfristig auch auf die Vermögensverteilung), sofern zusätzliches Sparen zustande kommt, gleich ob es sich um Produktivvermögen oder Geldvermögen etc. handelt. Von GB, die bar ausgezahlt werden, gehen keine eindeutigen dauerhaften Verteilungseffekte aus; je nach Ausgestaltung können sie für Arbeitnehmer positiv oder negativ sein. Dass die Umverteilung des Nationaleinkommens zulasten der Arbeitnehmer in den vergangenen Jahren durch GB umgekehrt werden kann, gilt nur, wenn Letztere on top, also zusätzlich gewährt werden. Gerade dies ist jedoch nicht die Intention der Protagonisten von GB. Insofern ist eine Beteiligung der Arbeitnehmer an „Globalisierungsgewinnen“ von den Unternehmen gar nicht beabsichtigt.

Ist eine stärkere staatliche Förderung von $\mathrm{GB} / \mathrm{KB}$ gerechtfertigt? Richtig ist, dass nur mit wesentlich stärkerer steuerlicher Begünstigung eine Kehrtwende bei $\mathrm{KB}$ erreicht werden könnte. ${ }^{7}$ GB könnten prob- lemlos ohne staatliche Förderung ausgeweitet werden, wenn die Tarifparteien dies wünschen (BDA/BDI 2007). Eine einseitige staatliche Begünstigung von $\mathrm{KB}$, insbesondere am arbeitgebenden Unternehmen, würde andere Vermögensanlagen diskriminieren und Arbeitnehmer zu riskanten Anlagen verleiten, die sie ohne staatliche Förderung vermeiden würden. Dies wäre nur dann ratsam, wenn andere, eindeutig positive Wirkungen entstünden, die ohne staatliche Förderung nicht einträten. Weder sind betriebliche Produktivitätswirkungen noch Beschäftigungseffekte sicher. Arbeitnehmerbeteiligung am Produktivvermögen ist auch kein Wert an sich. Die Beseitigung von Eigenkapitalmangel bei kleinen und mittleren Unternehmen, bei der sich private Kapitalgeber wegen Risiken und/ oder Intransparenz nicht engagieren, sollte nicht Arbeitnehmern aufgebürdet werden. Freilich gilt auch: Dort, wo sich Arbeitnehmer an „ihren“ Unternehmen beteiligen möchten und auch die Eigentümer einverstanden sind, jedoch administrative oder rechtliche Hemmnisse bestehen, sollten Erleichterungen erwogen werden. Dies gilt vor allem für Personengesellschaften.

Jüngst hat die CDU/CSU eine wesentlich stärkere steuerliche Förderung von $\mathrm{KB}$ am arbeitgebenden Unternehmen gefordert (CDU/CSU 2007). Für Arbeitnehmer ist Mitarbeiterbeteiligung (MAB) meist nur interessant, wenn es sich um florierende Unternehmen handelt und die Beteiligungen zu einem Vorzugspreis angeboten werden. Wenn Unternehmen Vorteile von $\mathrm{MAB}$ erwarten, werden sie nicht zögern, Gratifikationen anzubieten. Bedürfen sie staatlicher Unterstützungen, ist zu vermuten, dass die eigentlichen Vorteile gering sind und der Staat Fehlallokation subventioniert. Auch der von der SPD eingebrachte Vorschlag eines überbetrieblichen,

\footnotetext{
7 Derzeit fördert der Staat nach dem 5. Gesetz zur Förderung der Vermögensbildung der Arbeitnehmer mit der Arbeitnehmerzulage von max. $72 €$ p.a. (18\% der Sparsumme) - bei engen Einkommensgrenzen - im Umfang von 390 Mio. $€$ (2005). Dabei wird ein vorgegebenes Anlagespektrum gefördert; 2005 entfiel nur ein Drittel der Anlagen auf betriebliche und außerbetriebliche Beteiligungen. §19a EStG stellt von Arbeitgebern ihren Arbeitnehmern gewährte Kapitalbeteiligungen bis zu $135 €$ p.a. steuer- und abgabenfrei (Fördersumme insgesamt ca. 190 Mio. €). Entgeltumwandlung zur betrieblichen Altersvorsorge ist bis 2008 sozialabgabenfrei (Angaben nach SPD 2007)
} 
steuerlich geförderten Kapitalfonds, der in Unternehmensbeteiligungen investiert, überzeugt nicht (SPD 2007). Hier werden Anreize für Arbeitnehmer gesetzt, einseitig Anteile an Produktivvermögen zu erwerben. Zwar wird durch Beteiligungen an vielen Unternehmen eine gewisse Risikostreuung erreicht, aber es handelt sich gleichwohl um Anlagen in Risikokapital. Eine Förderung allgemeiner Vermögensbildung, insbesondere für untere Einkommens- schichten (und nicht nur für Arbeitnehmer), wäre sinnvoller. Vermögen ist ein wichtiger Teil sozialer Sicherheit. ${ }^{8}$ Zudem unterscheidet sich der von der SPD favorisierte "Deutschlandfonds" nicht wesentlich von privaten Aktienfonds. Eine spezielle steuerliche Förderung der Arbeitnehmerbeteiligung am Produktivvermögen ist überflüssig. Die Vorschläge beider Parteien sind insgesamt also schwer nachvollziehbar. Es scheint in erster Linie um die Wie- derbelebung alter Illusionen hinsichtlich der "Teilhabergesellschaft" unter dem neuen Etikett der „Sozialen Kapitalpartnerschaft" zu gehen - also um Ideologie, nicht Ökonomie.

Das unterste Zehntel der Haushalte besitzt keinerlei Vermögen, sondern nur Schulden (Krause/ Schäfer 2005, S. 200)

\section{LITERATUR}

\section{Arbeitsgemeinschaft Partnerschaft in der Wirtschaft (AGP) (2007): http://www.agpev.de/mitarbeiterbeteiligung/50905797a209e7604/ index.html (29.08.2007)}

Ammermüller, A./Weber, A. M./Westerheide, P. (2005): Die Entwicklung und Verteilung des Vermögens privater Haushalte unter besonderer Berücksichtigung des Produktivvermögens, ZEW, Mannheim BDA/BDI (2007): Mitarbeiterbeteiligung. Strategie für eine partnerschaftliche Unternehmenskultur, Berlin

Bellmann, L./Möller, I. (2006a): Gewinn- und Kapitalbeteiligung der Mitarbeiter. Die Betriebe in Deutschland haben Nachholbedarf, IAB-Kurzbericht 13, vom 5.9 .

Bellmann, L./Möller, I. (2006b): Produktivitätswirkungen der materiellen Mitarbeiterbeteiligung für das Unternehmen, in: Deutscher Gewerkschaftsbund (Hrsg.): Kapital in Mitarbeiterhand: Hemmschuh oder Motor für die Mitbestimmung? Dokumentation des Workshops am 16. Mai in Berlin, Berlin, S. 27-32

Blum, U. (2006): Produktivitäts- und Beschäftigungseffekte der Mitarbeiterbeteiligung, in: Wirtschaft im Wandel 9, S. 274-280

CDU/CSU (2007): Betriebliche Bündnisse für Soziale Kapitalpartnerschaften. Beschluss der gemeinsamen Arbeitsgruppe von CDU und CSU, Berlin Deutsche Rentenversicherung (DRV) (2005): Renten auf einen Blick: Staatliche Politik im OECD-Ländervergleich, DRV-Schriften 61, Frankfurt Deutsches Aktieninstitut (DAI) (2006): Aktionärszahlen rückläufig Steuerpolitik verunsichert Anleger, DAI-Kurzstudie 2, Frankfurt/M (www.dai.de)

Deutsches Aktieninstitut (DAI) (2007): DAI-Factbook, Stand 28.03.2007 (www.dai.de)

Gerlach, K. (1997): Beschäftigungsförderung durch Gewinnbeteiligung? in: Sadowski, D./Schneider, M. (Hrsg.): Vorschläge zu einer neuen Lohnpolitik. Optionen für mehr Beschäftigung, Bd. I, Frankfurt/M., New York, S. 311-329

Hardes, H.-D./Wickert, H. (2000): Erfolgsabhängige Beteiligungsentgelte in vergleichender Perspektive: Empirische Befunde und Erklärungsansätze, in: Zeitschrift für Personalforschung 14, S. 52-77

Hofmann, H./Holzner, C. (2002): Mitarbeiterbeteiligung - ein internationaler Vergleich, in: ifo-schnelldienst 12, S. 7-13

Krause, P./Schäfer, A. (2005): Verteilung von Vermögen und Einkommen in Deutschland: Große Unterschiede nach Geschlecht und Alter, in: DIWWochenbericht 11, S. 199-203
Oberhauser, A. (2006): Die verteilungstheoretischen Grundlagen des Investivlohns, in: Wirtschaftsdienst 2, S. 92-95

OECD (1995): Employment Outlook, Paris

Pendleton, A./Poutsma, E./v.Ommeren, J./Brewster, Ch. (2001): Employee Share Ownership and Profit-Sharing in the European Union, Dublin PEPPER (1991): Kommission der Europäischen Gemeinschaften, Der PEPPER-Bericht, in: Soziales Europa, Beiheft 3

PEPPER (1997): Europäisches Parlament, Bericht über den Bericht der Kommission über die Förderung der Gewinn- und Betriebsergebnisbeteiligung (einschließlich Kapitalbeteiligung) der Arbeitnehmer in den Mitgliedstaaten - PEPPER II - 1996, Sitzungsdokumente (30.9.), Brüssel Priewe, J. (2000): Mitarbeiterbeteiligung: Wohin führt der Weg? Optionen und Grenzen für die Zukunft, in: ISA-Consult (Hrsg.): Mitarbeiterbeteiligung als Instrument für Vermögenspolitik und Alterssicherung - Bilanz und Perspektiven, Berlin, S. 27-52

Priewe, J./Havighorst, F. (1999): Auf dem Weg zur Teilhabergesellschaft? Investivlöhne, Gewinn- und Kapitalbeteiligungen der Arbeitnehmer in Westeuropa und den USA - eine vergleichende Bestandsaufnahme, Friedrich-Ebert-Stiftung, Bonn

Schäfer, C./Rürup, B. (1998): Der Investivlohn. Förderung der Arbeitnehmer-Vermögensbildung? Möglichkeiten, Grenzen, Transformationen, Gutachten im Auftrag der Enquête-Kommission „Zukunft der Erwerbsarbeit" des Landtags von Nordrhein-Westfalen, Darmstadt und Düsseldorf Schnabel, C. (2004): Mitarbeiterbeteiligung - ein guter Weg zu höherer Produktivität und Flexibilität, in: Sozialer Fortschritt 4,S. 81-87 Sievert, O. (1992): Für Investivlöhne. Plädoyer für ein vernachlässigtes Konzept, Frankfurter Institut für wirtschaftspolitische Forschung, Bad Homburg

SPD (2007): Deutschlandfonds für Arbeitnehmerinnen und Arbeitnehmer, Berlin

Sprenger, B. (2007): Geldvermögen erreicht 4,5 Billionen Euro: Mehrheit der Deutschen verpasst die Aktienhausse, in: die bank, 9, Online Ausgabe, www. die-bank.de/print/Artikel.asp?art|D=568\&issue $=092007$ (02.09.2007)

Tofaute, H. (2006): Rückenwind für die Mitarbeiterbeteiligung durch die Tarifpartner? in: Wirtschaftsdienst 2, S. 79-84

Wolf, E./Zwick, T. (2002): Produktivitätsentwicklung von Mitarbeiterbeteiligungen: Der Einfluss von unbeobachteter Heterogenität, in: Mitteilungen aus der Arbeitsmarkt- und Berufsforschung 1, S. 123-132 